ANNALES

POLONICI MATHEMATICI

$93.3(2008)$

\title{
Geometry of Puiseux expansions
}

\author{
by Maciej Borodzik and Henryk ŻoŁĄDEK (Warszawa)
}

\begin{abstract}
We consider the space Curv of complex affine lines $t \mapsto(x, y)=(\phi(t), \psi(t))$ with monic polynomials $\phi, \psi$ of fixed degrees and a map Expan from Curv to a complex affine space Puis with dim Curv = dim Puis, which is defined by initial Puiseux coefficients of the Puiseux expansion of the curve at infinity. We present some unexpected relations between geometrical properties of the curves $(\phi, \psi)$ and singularities of the map Expan. For example, the curve $(\phi, \psi)$ has a cuspidal singularity iff it is a critical point of Expan. We calculate the geometric degree of Expan in the cases $\operatorname{gcd}(\operatorname{deg} \phi, \operatorname{deg} \psi) \leq 2$ and describe the non-properness set of Expan.
\end{abstract}

1. Introduction. Let $p, q$ be integers satisfying

$$
1<p<q, \quad q / p \notin \mathbb{Z} .
$$

We consider complex parametric curves $\xi: \mathbb{C} \rightarrow \mathbb{C}^{2}$ of the form

$$
x=\phi(t)=t^{p}+a_{1} t^{p-1}+\cdots+a_{p}, \quad y=\psi(t)=t^{q}+b_{1} t^{q-1}+\cdots+b_{q},
$$

with

$$
a_{1}=0 .
$$

Denote by $C=\xi(\mathbb{C})$ the image of the curve $\xi$. The curve $C$ has the Puiseux expansion at infinity

$$
y=x^{q / p}+c_{1} x^{(q-1) / p}+\cdots,
$$

where $c_{j}=c_{j}(\xi)=c_{j}(a, b)$ are polynomials in $(a, b)=\left(a_{2}, \ldots, a_{p}, b_{1}, \ldots, b_{q}\right)$; they are uniquely defined by the condition $x^{1 / p}=t(1+o(1))$ as $t \rightarrow \infty$. Any polynomial curve in $\mathbb{C}^{2}$ can be reduced either to a curve of the form (1.2) with conditions (1.1) and (1.3), or to a straight line $t \mapsto(t, 0)$ by a Cremona automorphism of the plane and a shift of the time $t$.

2000 Mathematics Subject Classification: Primary 14H50; Secondary 14H20, 14H45, $14 \mathrm{~B} 05$.

Key words and phrases: Puiseux expansion, affine algebraic curve.

Supported by Polish KBN Grant No 1 PO3A 01529. 
Definition 1.1. We define the space of curves $\operatorname{Curv}=\operatorname{Curv}_{p, q} \simeq$ $\mathbb{C}^{p+q-1}$ as the space consisting of the parametric lines (1.2). The space Puis $=$ Puis $_{p, q} \simeq \mathbb{C}^{p+q-1}$ is the space of truncated Puiseux series $y=$ $x^{q / p}+c_{1} x^{(q-1) / p}+\cdots+c_{p+q-1} x^{(1-p) / p}$. The map

$$
\xi \mapsto\left(c_{1}(\xi), \ldots, c_{p+q-1}(\xi)\right)
$$

is called the expansion map and denoted by $\operatorname{Expan}=\operatorname{Expan}_{p, q}$.

The expansion map is the main subject of this paper. It can be regarded as a certain generalisation of the Lyashko-Looijenga map. Recall that the Lyashko-Looijenga map associates to any meromorphic function on $\mathbb{C P}^{1}$ its unordered collection of critical values and is used in the Hurwitz problem of ramified covering of the sphere $S^{2}$ (see [Lan]). Here we have two rational functions, but of a special form, and we study one critical point, but in greater detail.

We use the map Expan to study the singularities of the curve. The idea to consider this map has arisen in our investigations of polynomial curves with $b^{1}=1$ in [BZ1] and of the Jacobian conjecture [Zol].

Definition 1.2. A curve $\xi=(\phi, \psi)$ is singular at $t_{0}$ if $\dot{\phi}\left(t_{0}\right)=\dot{\psi}\left(t_{0}\right)=0$, where the dot denotes the derivation with respect to $t$. A curve is singular if it is singular at some point.

REMARK 1.3. An intersection of smooth branches of a curve $C$, even with tangency, is not regarded as a singularity in this paper.

Our first result connects singularities of curves with singularities of the expansion map.

Theorem 1.4. A curve $\xi_{0} \in \mathrm{Curv}$ is a critical point of Expan if and only if $\xi_{0}$ is a singular curve. Moreover, the multiplicity of Expan at $\xi_{0}$ is infinite iff the parametrisation $t \mapsto \xi(t)$ is not one-to-one.

Theorem 1.5. Assume that $\xi_{0}$ has a single singular point of type $\mathbf{A}_{2 m}$. Then the multiplicity of Expan at $\xi_{0}$ is $m+1$. Moreover, if $2 m+1 \leq q$, the map Expan has the Morin singularity at that point.

Recall that the $\mathbf{A}_{2 k}$ singularity of a curve, or of a function, is defined as $x^{2}+y^{k+1}=0$, and the Morin singularity of multiplicity $k$ is given by the following normal form:

$$
\left(s, \lambda_{1}, \ldots, \lambda_{n-1}\right) \mapsto\left(s^{k}+\lambda_{1} s^{k-2}+\cdots+\lambda_{k-2} s, \lambda_{1}, \ldots, \lambda_{n-1}\right) .
$$

Theorems 1.4 and 1.5 are proved in the next section.

Definition 1.6. A continuous map $G: X \rightarrow Y$ is proper if the preimage of any compact subset of $Y$ is compact. The set of points $y_{0} \in Y$ such that for any neighbourhood $U \subset Y$ with compact closure $\bar{U}$ the inverse image $G^{-1}(\bar{U})$ is not compact, is called the non-properness set $S(G)$. In other 
words, any $y_{0} \in S(G)$ can be approximated by points $y_{n} \in Y$ such that there exists a sequence $x_{n} \in X$ with $G\left(x_{n}\right)=y_{n}$ such that $x_{n}$ does not have a convergent subsequence.

We define

$$
p^{\prime}=\operatorname{gcd}(p, q), \quad p_{1}=\frac{p}{p^{\prime}}, \quad q_{1}=\frac{q}{p^{\prime}} .
$$

Theorem 1.7. (a) If $p^{\prime}=1$ then the map Expan is a proper ramified covering of topological degree

$$
\operatorname{deg}_{\text {top }} \operatorname{Expan}_{p, q}=\frac{(p+q-1) !}{p ! q !} .
$$

(b) If $p^{\prime}>1$ then the non-properness set is

$$
S(\text { Expan })=\left\{c_{1}=0\right\} .
$$

(c) If $p^{\prime}=2$ then

$$
\operatorname{deg}_{\text {top }} \operatorname{Expan}=\frac{(p+q-1) !}{p ! q !}-\frac{1}{2}\left(\frac{\left(p_{1}+q_{1}-1\right) !}{p_{1} ! q_{1} !}\right)^{2} .
$$

This theorem is proved in the third section.

We finish this introduction by remarking that the map Expan can be generalised to other types of plane curves. Namely, to affine curves of fixed genus and fixed orders of poles (or multiplicities of parametrisation at finite singularities) one can associate a finite number of Puiseux coefficients of the expansions at the places at infinity (or at singularities). We plan to continue this study in further works.

2. Local singularities. Let $\xi_{0}=\left(\phi_{0}, \psi_{0}\right) \in$ Curv. We would like to

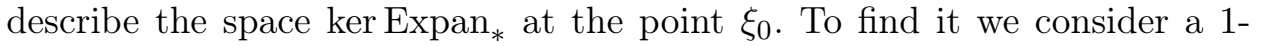
parameter first order perturbation of $\xi_{0}$,

$$
\xi^{s}(t)=\left(\phi^{s}(t), \psi^{s}(t)\right)=\left(\phi_{0}+s \phi_{1}, \psi_{0}+s \psi_{1}\right)=\xi_{0}+s \xi_{1},
$$

where the map $\xi_{1}=\left(\phi_{1}, \psi_{1}\right)$ is polynomial and satisfies

$$
\operatorname{deg} \phi_{1} \leq p-2, \quad \operatorname{deg} \psi_{1} \leq q-1,
$$

and can be regarded as an element of $T_{\xi_{0}}$ Curv. Then $\xi_{1} \in \operatorname{ker} \operatorname{Expan}_{*}\left(\xi_{0}\right)$ iff $\operatorname{Expan}\left(\xi^{s}\right)-\operatorname{Expan}\left(\xi_{0}\right)=O\left(s^{2}\right)$, i.e.

$$
c_{j}(s)=c_{j}(0)+O\left(s^{2}\right), \quad j=1, \ldots, p+q-1, \quad \text { as } s \rightarrow 0 .
$$

Let $P: \mathbb{C}^{2} \rightarrow \mathbb{C}^{2}$ be a polynomial map defined by

$$
P(t, s)=\xi^{s}(t) .
$$

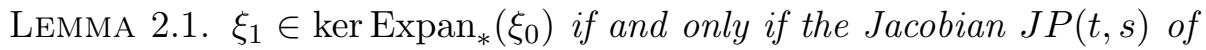
the map $P$ vanishes identically for $s=0$. 
Proof. Consider the expansions

$$
\left\{\begin{array}{l}
\phi^{s}(t)=a_{0}(s) t^{p}+a_{1}(s) t^{p-1}+\cdots \\
\psi^{s}(t)=b_{0}(s) t^{q}+b_{1}(s) t^{q-1}+\cdots
\end{array}\right.
$$

where $a_{0} \equiv b_{0} \equiv 1$. The expansion of $J P$ in powers of $t$ gives

$$
J P=t^{p+q-1}\left|\begin{array}{cc}
p a_{0} & a_{0}^{\prime} \\
q b_{0} & b_{0}^{\prime}
\end{array}\right|+\cdots=0 \cdot t^{p+q-1}+\cdots .
$$

We perform the formal change

$$
\psi^{s}(t) \mapsto \psi_{1}^{s}(t)=\psi^{s}(t)-\left[\phi^{s}(t)\right]^{q / p},
$$

where $\left[\phi^{s}(t)\right]^{q / p}=t^{q}\left(1+\frac{q}{p} t^{-1}+\cdots\right)$ is a series with coefficients polynomial in $s$. We have $\psi_{1}^{s}=c_{1}(s) t^{q+p-1}+\cdots$. Moreover,

$$
J P=J\left(\phi^{s}(t), \psi_{1}^{s}(t)\right)=p c_{1}^{\prime}(s) t^{p+q-2}+\cdots .
$$

We see that $c_{1}^{\prime}(0)=0$ if and only if $J P(t, 0)$ has degree less than $p+q-2$.

Assuming the latter, we apply the change $\psi_{1}^{s} \mapsto \psi_{2}^{s}=\psi_{1}^{s}-c_{1}(s)\left[\phi^{s}\right]^{(q-1) / p}$, which has the property that $J\left(\phi^{s}, \psi_{2}^{s}\right)=J P+O(s)$ (because $c_{1}(s)-c_{1}(0)=$ $\left.O\left(s^{2}\right)\right)$. We have $\psi_{2}^{s}=c_{2}(s) t^{p+q-3}+\cdots$, so again we obtain

$$
c_{2}^{\prime}(0)=0 \Leftrightarrow \operatorname{deg}_{t} J P(t, 0)<p+q-3,
$$

under the assumption $c_{1}^{\prime}(0)=0$. Repeating this procedure we obtain

$$
c_{j}^{\prime}(0)=0, j=1, \ldots, p+q-1 \Leftrightarrow \operatorname{deg}_{t} J P(t, 0)<0 .
$$

Lemma 2.1 admits a generalisation to higher order deformations of the form

$$
\xi^{s}=\xi_{0}+s \xi_{1}+\cdots+s^{k} \xi_{k},
$$

with $\xi_{j}=\left(\phi_{j}, \psi_{j}\right)$ satisfying $(2.2)$. We define the polynomial map $P$ as in (2.4) with $\xi^{s}$ given by $(2.10)$.

Lemma 2.2. The Puiseux coefficients $c_{j}(s), j=1, \ldots, p+q-1$, of the curve $\xi^{s}$ have the property

$$
c_{j}(s)=c_{j}(0)+O\left(s^{k+1}\right)
$$

if and only if

$$
J P(t, s)=O\left(s^{k}\right) .
$$

Proof. This proof repeats the proof of Lemma 2.1. Under the assumption that $c_{1}(s)-c_{1}(0), \ldots, c_{j}(s)-c_{j}(0)$ are $O\left(s^{k+1}\right)$, the formal change $\psi_{j+1}^{s}=$ $\psi_{j}^{s}-c_{j}(s)\left[\phi^{s}\right]^{(q-j) / p}$ has the property that

$$
J\left(\phi^{s}, \psi_{j+1}^{s}\right)=J\left(\phi^{s}, \psi_{j}^{s}\right)+O\left(s^{k}\right) .
$$

Hence $c_{j}^{\prime}=O\left(s^{k}\right)$ for $j=1, \ldots, p+q-1$ if and only if $\left.\operatorname{deg}_{t} \frac{\partial^{i}}{\partial s^{i}} J(t, s)\right|_{s=0}<0$ for $i=0, \ldots, k=1$. 
The above two lemmas have very interesting consequences, the first being the proof of Theorem 1.4 given below.

Proof of Theorem 1.4. A point $\xi_{0} \in$ Curv is a critical point for the map Expan iff there exists a non-trivial element $\left(\phi_{1}, \psi_{1}\right) \in \operatorname{ker} \operatorname{Expan}_{*}\left(\xi_{0}\right)$. By Lemma 2.1 and formula (2.2) we should have

$$
\left|\begin{array}{cc}
\dot{\phi}_{0} & \phi_{1} \\
\dot{\psi}_{0} & \psi_{1}
\end{array}\right| \equiv 0 .
$$

This means that

$$
\frac{\phi_{1}}{\psi_{1}}(t)=\frac{\dot{\phi}_{0}}{\dot{\psi}_{0}}(t)
$$

(identity of rational functions). Recall that $\operatorname{deg} \dot{\phi}_{0}=p-1$ and $\operatorname{deg} \dot{\psi}_{0}=$ $q-1$. If $\dot{\phi}_{0}$ and $\dot{\psi}_{0}$ are coprime then any solution to (2.11) has the form $\phi_{1}=\chi(t) \dot{\phi}_{0}(t), \psi_{1}=\chi(t) \dot{\psi}_{0}(t)$, where $\chi$ is a polynomial. But comparison of degrees shows that $\chi \equiv 0$, i.e. $\operatorname{ker} \operatorname{Expan}_{*}\left(\xi_{0}\right)=0$.

Conversely, if $\operatorname{gcd}\left(\dot{\phi}_{0}, \dot{\psi}_{0}\right)=\eta(t)$ with $\operatorname{deg} \eta>0$, then for any polynomial $\eta_{1}$ dividing $\eta$ such that $\operatorname{deg} \eta_{1}>0$, the polynomials $\phi_{1}=\dot{\phi}_{0} / \eta_{1}, \psi_{1}=\dot{\psi}_{0} / \eta_{1}$ satisfy (2.11) and the degree bounds (2.2), so they provide an element of ker $\operatorname{Expan}_{*}\left(\xi_{0}\right)$. It is easy to see that all solutions of (2.11) are obtained in this way, therefore we get the formula

$$
\operatorname{dim} \operatorname{ker} \operatorname{Expan}_{*}\left(\xi_{0}\right)=\operatorname{deg} \operatorname{gcd}\left(\dot{\phi}_{0}, \dot{\psi}_{0}\right) .
$$

The second statement of Theorem 1.4 says that the multiplicity of Expan at $\xi_{0}$ is infinite iff the curve $\xi_{0}$ is multiply covered. Recall that the multiplicity of Expan at $\xi_{0}$ is equal to the supremum of the number of preimages near $\xi_{0}$ of points near $\operatorname{Expan}\left(\xi_{0}\right)[\mathrm{AVG}]$. Moreover, $\mu_{\xi_{0}} \operatorname{Expan}=\infty$ iff there exists a germ of non-constant curve at $\xi_{0}$ which is sent to one point. On the other hand, the multiply covered curves have the form

$$
\xi(t)=\tilde{\xi} \circ \omega(t),
$$

where $\omega$ is a polynomial of degree $d>1$ and $\tilde{\xi}$ is a polynomial curve (the Lüroth theorem). Of course, in that case $d \mid \operatorname{gcd}(p, q)$.

It is clear that the curves of the form $\widetilde{\xi} \circ \omega$ with $\operatorname{deg} \omega>1$ represent non-isolated critical points of the map Expan: we can vary coefficients of $\omega$ without changing the Puiseux coefficients $c_{j}$.

Assume that $\xi_{0}$ is a non-isolated critical point of Expan. Take a germ of non-constant curve $(\mathbb{C}, 0) \supset(U, 0) \rightarrow\left(\right.$ Curv, $\left.\xi_{0}\right), s \mapsto \xi^{s}$, which is sent to one point $\operatorname{Expan}\left(\xi_{0}\right) \in$ Puis. Define a holomorphic mapping $P: U \times \mathbb{C}$ $\rightarrow \mathbb{C}^{2}:(t, s) \mapsto \xi^{s}(t)$. By passing to the limit as $k \rightarrow \infty$ in Lemma 2.2 we find that the conditions $c_{1}(s) \equiv c_{1}(0), \ldots, c_{p+q-1}(s) \equiv c_{p+q-1}(0)$ are 
equivalent to $J P \equiv 0$. But this means that the image of $P$ is a single curve, $P(U \times \mathbb{C})=C_{0}=\xi_{0}(\mathbb{C})$. In particular, $\xi^{s}(\mathbb{C})=C_{0}$ for any $s \in U$.

The supposition that $\xi_{0}$ is primitive leads to the conclusion that the elements $\xi^{s}$ define different parametrisations of one rational curve $C_{0}$. Each such parametrisation defines an automorphism of $\mathbb{C}$, which is of the form $t \mapsto t+a$. But the condition $a_{1}=0$ (see (1.3)) means that $a=0$. So $\xi^{s}(t) \equiv \xi_{0}(t)$, which contradicts the assumption that the germ $s \mapsto \xi^{s}$ is non-constant.

COROllary 2.3. The determinant det $\operatorname{Expan}_{*}\left(\xi_{0}\right)$ is proportional to the resultant of $\dot{\phi}_{0}$ and $\dot{\psi}_{0}$.

Proof. Theorem 1.4 shows that the zero sets of $\operatorname{det} \operatorname{Expan}_{*}\left(\xi_{0}\right)$ and of the resultant are the same. Since the resultant is reduced, $\operatorname{det} \operatorname{Expan}_{*}\left(\xi_{0}\right)$ is a power of the resultant. An easy comparison of degrees shows that it is in fact proportional.

Proof of Theorem 1.5. Consider first the situation when

$$
x=\phi_{0}(t)=\left(t-t_{0}\right)^{2}+\cdots, \quad y=\psi_{0}(t)=\left(t-t_{0}\right)^{2 m+1}+\cdots,
$$

and we put $t_{0}=0$ to simplify the notation. The assumption implies that the polynomials $\dot{\phi}_{0} / t$ and $\dot{\psi}_{0} / t$ are relatively prime. We shall construct a deformation

$$
\xi^{s}(t)=\xi_{0}(t)+s \xi_{1}(t)+\cdots+s^{m+1} \xi_{m+1}(t),
$$

where $\xi_{1} \in \operatorname{ker} \operatorname{Expan}_{*}\left(\xi_{0}\right) \backslash 0$, such that

$$
\begin{aligned}
c_{j}(s) & =c_{j}(0)+O\left(s^{m+2}\right), \quad j=1, \ldots, p+q-2, \\
c_{p+q-1}(s) & =c_{p+q-1}(0)+\nu s^{m+1}+O\left(s^{m+2}\right), \quad \nu \neq 0 .
\end{aligned}
$$

By Lemma 2.2 we should calculate the Jacobian of the 2-dimensional map $P$ associated with the deformation (2.15). We have the expansion $J P=$ $J_{0}(t)+s J_{1}(t)+\cdots+s^{k} J_{k}(t)+\cdots$, where

$$
J_{j}(t)=(j+1)\left|\begin{array}{cc}
\dot{\phi}_{0} & \phi_{j+1} \\
\dot{\psi}_{0} & \psi_{j+1}
\end{array}\right|+\cdots+\left|\begin{array}{cc}
\dot{\phi}_{j} & \phi_{1} \\
\dot{\psi}_{j} & \psi_{0}
\end{array}\right| .
$$

The deformation (2.15) satisfies (2.16) iff

$$
\begin{aligned}
J_{j} & \equiv 0 \quad \text { for } j=0, \ldots, m-1, \\
J_{m} & =\text { const. }
\end{aligned}
$$

The construction of the deformation (2.15) relies on solving the equation (2.18) inductively. Namely, for given $j$ the equation (2.18) can be regarded as an equation defining $\xi_{j+1}=\left(\phi_{j+1}, \psi_{j+1}\right)$ with $\xi_{i}$ for $i \leq j$ already known. The details are the following. First, let $\phi_{1}=\dot{\phi}_{0} / t=2+\cdots, \psi_{1}=\dot{\psi}_{0} / t=$ $(2 m+1) t^{2 m-1}+\cdots$. Note that $\operatorname{deg} \phi_{1}=p-2$ and $\operatorname{deg} \psi_{1}=q-2$. The 
equation $J_{1} \equiv 0$ has the form

$$
2\left|\begin{array}{cc}
\dot{\phi}_{0} & \phi_{2} \\
\dot{\psi}_{0} & \psi_{2}
\end{array}\right|=\dot{\phi}_{1} \psi_{1}-\dot{\psi}_{1} \phi_{1}=2\left(4 m^{2}-1\right) t^{2 m-2}+\cdots .
$$

After dividing this equation by $2 t$ we get

$$
\psi_{2} \phi_{1}-\phi_{2} \psi_{1}=q_{2}(t),
$$

where $q_{2}$ is a polynomial of degree $\leq p+q-5$ and $\operatorname{ord}_{t=0} q_{2}(t)=2 m-3$. Since $\phi_{1}$ and $\psi_{1}$ are relatively prime, there exists a unique solution modulo $\left(\phi_{1}, \psi_{1}\right)$ such that $\operatorname{deg} \phi_{2} \leq p-2$ and $\operatorname{deg} \psi_{2} \leq q-2$ (see [War, §36]). Moreover, we find that $\psi_{2}=c t^{2 m-3}+\cdots$ as $t \rightarrow 0$, with $c \neq 0$ being a constant.

By induction we show that the equations $J_{j} \equiv 0$ for $j=0, \ldots, m-1$ have solutions $\phi_{j+1}, \psi_{j+1}$ such that $\operatorname{deg} \phi_{j+1} \leq p-2, \operatorname{deg} \psi_{j+1} \leq q-2$ and $\psi_{j+1} \sim t^{2 m-2 j-1}$ around $t=0$.

For $j=m$ we find that $J_{m}=(m+1) t\left(\phi_{1} \psi_{m+1}-\psi_{1} \phi_{m+1}\right)-q_{m+1}(t)$, where $q_{m+1}=\phi_{1} \dot{\psi}_{m}+\cdots=\kappa+O(t)$ with $\kappa \neq 0$. Therefore the equation $J_{m} \equiv 0$ has no solution. However, we can find $\left(\phi_{m+1}, \psi_{m+1}\right)$ such that $J_{m}=-\kappa /(m+1)$, so $J P=-\frac{\kappa}{m+1} s^{m}+O\left(s^{m+1}\right)$.

The deformation (2.15) is unique in a sense. The only non-uniqueness in solving $J_{j} \equiv 0$ means adding a scalar multiple of $\xi_{1}$ to $\xi_{j+1}$, which corresponds to reparametrisations of parameter $s$. This non-uniqueness does not influence the leading term in $\psi_{j+1}$.

We can interpret this result as follows:

Lemma 2.4. The maximal order $\operatorname{ord}_{s=0} \operatorname{Expan}\left(\xi^{s}\right)$ over all curve germs $(\mathbb{C}, 0) \rightarrow\left(\mathrm{Curv}, \xi_{0}\right)$ with $\left.\frac{d}{d s} \xi^{s}\right|_{s=0} \neq 0$ equals $m+1$.

Now we consider the general case, i.e. without assuming (2.14). We still use $t_{0}=0$ to simplify the notation. So we have either

$$
\phi_{0}=t^{2}+\cdots, \quad \psi_{0}=\beta_{2 r} t^{2 r}+\cdots,
$$

or

$$
\phi_{0}=\alpha_{2 r} t^{2 r}+\cdots, \quad \psi_{0}=t^{2}+\cdots ;
$$

so there exists a change of variables (in the target plane) either of the form

$$
\begin{aligned}
\psi_{0} \mapsto \widetilde{\psi}_{0} & =\psi_{0}-c_{2 r}^{(0)} \phi_{0}^{2 r}-c_{2 r+2}^{(0)} \phi_{0}^{2 r+2}-\cdots-c_{2 m}^{(0)} \phi_{0}^{m} \\
& =c_{2 m+1}^{(0)} t^{2 m+1}+\cdots
\end{aligned}
$$

or of the form

$$
\begin{aligned}
\phi_{0} \mapsto \widetilde{\phi}_{0} & =\phi_{0}-d_{2 r}^{(0)} \psi_{0}^{2 r}-d_{2 r+2}^{(0)} \psi_{0}^{2 r+2}-\cdots-d_{2 m}^{(0)} \psi_{0}^{m} \\
& =d_{2 m+1}^{(0)} t^{2 m+1}+\cdots,
\end{aligned}
$$


respectively. It is obvious that in both cases the Puiseux coefficients $c_{1}^{(0)}$, $c_{3}^{(0)}, \ldots, c_{2 m-1}^{(0)}\left(d_{1}^{(0)}, d_{3}^{(0)}, \ldots, d_{2 m-1}^{(0)}\right)$ in $(2.22)$ (respectively $(2.23)$ ) form part of a local coordinate system near $\xi_{0}$ as long as $2 m+1 \leq q=\operatorname{deg} \psi$ (or $2 m+1 \leq p-1)$.

Lemma 2.5. In the general case, the maximal order $\operatorname{ord}_{s=0} \operatorname{Expan}\left(\xi^{s}\right)$ over all curve germs $(\mathbb{C}, 0) \rightarrow\left(\mathrm{Curv}, \xi_{0}\right)$ with $\left.\frac{d}{d s} \xi^{s}\right|_{s=0} \neq 0$ equals $m+1$.

Proof. Consider the curve $\xi_{0}=\left(\phi_{0}, \widetilde{\psi}_{0}\right)$ assuming the form (2.20) (the case (2.21) is analogous). Then $\xi_{0}$ is in $\operatorname{Curv}_{p, q}$ if $m p<q$ or in $\operatorname{Curv}_{p, \widetilde{q}}$ with $\widetilde{q}=p m$ otherwise. The first case is straightforward, so let $m p>q$. Denote by $\widetilde{c}_{j}$ the coefficients of the Puiseux expansion of $\widetilde{\psi}_{0}$ in powers of $\phi_{0}$ at infinity. By the proof of Lemma 2.4 there exists a deformation

$$
\begin{aligned}
& \phi^{s}=\phi_{0}+s \phi_{1}+\cdots+s^{m+1} \phi_{m+1}, \\
& \widetilde{\psi}^{s}=\widetilde{\psi}_{0}+s \widetilde{\psi}_{1}+\cdots+s^{m+1} \widetilde{\psi}_{m+1}
\end{aligned}
$$

such that $\widetilde{c}_{j}(s)-\widetilde{c}_{j}(0)=O\left(s^{m+2}\right)$ for $j=1, \ldots, p+\widetilde{q}-2, \widetilde{c}_{p+\widetilde{q}-1}(s)-$ $\widetilde{c}_{p+\widetilde{q}-1}(0)=$ const $\cdot s^{m+1}+O\left(s^{m+2}\right)$. We apply the inverse change to $(2.22)$, thus we put

$$
\psi^{s}=\widetilde{\psi}^{s}+c_{2 r}^{(0)} \cdot\left(\phi^{s}\right)^{2 r}+\cdots+c_{2 m}^{(0)} \cdot\left(\phi^{s}\right)^{2 m} .
$$

Then $\psi^{s}=\psi_{0}+s \psi_{1}+\cdots+s^{m+1} \psi_{m+1}+\cdots$. This will be our deformation, once we have shown that $\operatorname{deg} \psi_{j}(t) \leq q-1$.

Let $d=\max \left(\operatorname{deg} \psi_{j}: j=1, \ldots, m\right)$. Assume $d \geq q$. Let $j_{0}$ be the smallest non-zero integer such that $\operatorname{deg} \psi_{j_{0}}=d$. The Puiseux expansion of the curve $x=\phi^{s}, y=\psi^{s}$ is of the form

$$
y=-\widetilde{c}_{2 m}^{(0)}(s) x^{m}-\cdots-\widetilde{c}_{2 \widetilde{r}}^{(0)}(s) x^{\widetilde{r}}+\widetilde{c}_{\widetilde{q}-d}(s) x^{d / p}+\cdots
$$

for some $\widetilde{r}$. On the one hand, $\widetilde{c}_{\widetilde{q}-d}(s)=\widetilde{c}_{\widetilde{q}-d}(0)+O\left(s^{m+1}\right)$. On the other hand, $\widetilde{c}_{\widetilde{q}-d}(s)=\widetilde{c}_{\widetilde{q}-d}(0)+$ const $\cdot s^{j_{0}}$, and $j_{0} \leq m$. The contradiction shows that $d \leq q-1$.

Now we pass to the determination of the singularity of the map Expan at $\xi_{0}$, under the hypothesis that $\xi_{0}$ has a single singular point of type $\mathbf{A}_{2 m}$. By (2.12) the kernel of $\operatorname{Expan}_{*}\left(\xi_{0}\right)$ is one-dimensional, so the singular point is of corank 1 . Moreover, there exist local coordinates $(z, \lambda) \in\left(\mathbb{C} \times \mathbb{C}^{p+q-2}, 0\right)$ in the ambient space and $(w, \mu) \in\left(\mathbb{C} \times \mathbb{C}^{p+q-2}, 0\right)$ in the target space such that the map Expan near the point $\xi_{0}$ has the form (see [AVG])

$$
w=f(z, \lambda), \quad \mu=\lambda .
$$

The 1-dimensional map $z \mapsto w=f(z, 0)$ is called the genotype of (2.25). By [Mor] the genotype of a map of corank 1 is equivalent to

$$
z \mapsto z^{k+1}
$$

for some $k$. 
LEMMA 2.6. $k=m$.

Proof. This follows from Lemma 2.5.

Corollary 2.7. The multiplicity of Expan at $\xi_{0}$ equals

$$
\mu_{\xi_{0}} \operatorname{Expan}=m+1 \text {. }
$$

The function $f$ is a deformation of (2.26); by the Weierstrass preparation theorem we can write

$$
f(z, \lambda)=z^{m+1}+\alpha_{1}(\lambda) z^{m-1}+\cdots+\alpha_{m-1}(\lambda) z_{0},
$$

where the $\alpha_{j}(\lambda)$ are analytic.

Now we will show that if $2 m+1 \leq q$, the functions $\alpha_{1}(\lambda), \ldots, \alpha_{m-1}(\lambda)$ form part of a coordinate system near $\xi_{0}$. For curves $\xi$ near $\xi_{0}$ we can fix the critical point of $\phi$ at $t_{0}=0$. Thus we have

$$
x=\phi(t)=t^{2}+\cdots, \quad y=\psi(t)=\beta_{1} t+\cdots+\beta_{q} t^{q} .
$$

The Puiseux expansion of $C=\xi(\mathbb{C})$ at $t=0$ takes the form

$$
y=c_{1}^{(0)} x^{1 / 2}+c_{2}^{(0)} x+\cdots .
$$

Assume now $2 m+1 \leq q$. Then it is clear that the coefficients

$$
c_{1}^{(0)}, c_{3}^{(0)}, \ldots, c_{2 m-1}^{(0)}
$$

form part of a coordinate system in (Curv, $\left.\xi_{0}\right)$, the coefficient $c_{i}^{(0)}$ being linear in $\beta_{i}$.

Define the varieties near $\xi_{0}$

$$
\Sigma_{k+1}=\left\{\xi: c_{1}^{(0)}=\cdots=c_{2 k-1}^{(0)}=0\right\} .
$$

Repeating the proof of Lemma 2.4 one can show that they are Boardman's varieties $\Sigma^{1, \ldots, 1}$ defined as follows (see $[\mathrm{AVG}]$ ):

$$
\begin{aligned}
\Sigma^{1} & =\left\{\xi: \operatorname{dim} \operatorname{ker} \operatorname{Expan}_{*}(\xi)=1\right\}, \\
\Sigma^{1,1} & =\left\{\xi \in \Sigma^{1}: \operatorname{dim} \operatorname{ker}\left(\left.\operatorname{Expan}\right|_{\Sigma^{1}}\right)_{*}(\xi)=1\right\}, \\
& \vdots \\
\Sigma^{1, \ldots, 1} & =\left\{\xi \in \Sigma^{1, \ldots, 1}: \operatorname{dim} \operatorname{ker}\left(\left.\operatorname{Expan}\right|_{\Sigma^{1, \ldots, 1}} ^{k-1}\right)_{*}(\xi)=1\right\} .
\end{aligned}
$$

Our assumption $2 m+1 \leq q$ implies that $\Sigma_{k+1}$ is a smooth variety of codimension $k$.

In terms of the form (2.28) we have

$$
\Sigma^{1, \ldots, 1}=\left\{(z, \lambda): f_{z}^{\prime}=f_{z}^{\prime \prime}=\cdots=f_{z}^{(k)}=0\right\} .
$$


Hence $\alpha_{1}(\lambda), \ldots, \alpha_{m-1}(\lambda)$ in (2.28) form part of a local coordinate system near $\xi_{0}$. This proves that for $2 m+1 \leq q$ the map Expan has the Morin singularity. The proof of Theorem 1.5 is now finished.

REMARK 2.8. If $2 m+1>q$, the coefficients $c_{1}^{(0)}, c_{3}^{(0)}, \ldots, c_{2 m-1}^{(0)}$ do not necessarily form part of a local coordinate system in $\operatorname{Curv}_{p, q}$. The sets $\Sigma^{1, \ldots, 1}$ and $\Sigma^{k+1}$ are still identical, but do not have to be smooth. In [BZ2] we we study the regularity properties of the sequences formed by these Puiseux coefficients in Curv near a given point, namely the space defined by $c_{1}^{(0)}=$ $c_{3}^{(0)}=\cdots=c_{2 k+1}^{(0)}=0$ should have codimension $k$ in Curv or be empty.

ExAmple 2.9. Consider the map $\operatorname{Expan}_{3,4}$. Let us find its critical points. Using the change $\psi \mapsto \psi-$ const $\cdot \phi$ we can assume that $\phi=t^{3}+3 a t+3 b$, $\psi=t^{4}+c t^{2}+d t+e$ and $c_{1}=0$. Then $c_{2}=c-4 a, c_{3}=d-4 b, c_{4}=$ $e-2 a^{2}-2 a c_{2}, c_{5}=-4 a b-2 b c_{2}-a c_{3}, c_{6}=\frac{4}{3} a^{3}-2 b^{2}+a^{2} c_{2}-b c_{3}$. The vector $v=A \partial_{a}+B \partial_{b}+C \partial_{c}+D \partial_{d}+E \partial_{e}$ belongs to ker $\operatorname{Expan}_{*}$ if it satisfies $C=4 A, D=4 B, E=2\left(2 a+c_{2}\right) A, 0=-\left(4 b+c_{3}\right) A-2\left(2 a+c_{2}\right) B$, $0=2\left(2 a+c_{2}\right) A-\left(4 b+c_{3}\right) B$. These equations imply that ker $\operatorname{Expan}_{*}$ is non-trivial if and only if

$$
g=\left(4 b+c_{3}\right)^{2}+4 a\left(2 a+c_{2}\right)^{2}=0 .
$$

On the other hand, the condition $\dot{\xi}\left(t_{0}\right)=0$ implies $t_{0}^{2}+a=4 t_{0}^{3}+2 c t_{0}+d=0$. Elimination of $t_{0}$ gives $d^{2}+4 a(c-2 a)^{2}=0$, which coincides with (2.29) and is proportional to the resultant of $\dot{x}$ and $\dot{y}$.

To find $\Sigma^{1,1}$ we add the equation $d g(v)=0$. Since $d c_{j}(v)=0$, we get

$$
4\left(c_{2}^{2}+8 a c_{2}+12 a^{2}\right) A+8\left(4 b+c_{3}\right) B=0,
$$

which leads to the equation $c_{2}^{2}+12 a c_{2}+20 a^{2}=0$. We get two solutions: (i) $c_{2}=-2 a$, (ii) $c_{2}=-10 a$.

The case (i) implies $c=-2 t_{0}^{2}, d=0$ and $a=-t_{0}^{2}$. The curve has two singular points and ker $\operatorname{Expan}_{*}$ is 2-dimensional, so this is not an element of $\Sigma^{1,1}$.

In the case (ii) we find $c=6 t_{0}^{2}, d=-16 t_{0}^{3}$ and $a=-t_{0}^{2}$, which means the singularity $\mathbf{A}_{4}$. Therefore the set of curves with singularity $\mathbf{A}_{4}$ coincides with $\Sigma^{1,1}$.

The case of multiple singular points or one singular point with multiplicity $m>2$ is more difficult to handle. One can, at least in the case of one singular point, try to define an $(m-1)$-parameter deformation $\xi=\xi_{0}+\sum_{I} s^{I} \xi_{I}$ (with $s=\left(s_{1}, \ldots, s_{m-1}\right)$ and $I$ a multiindex) such that $c_{p+q-1}, c_{p+q-2}, \ldots$ $\ldots, c_{p+q+1-m}$ are of fixed order in $s_{1}, \ldots, s_{m-1}$, strictly greater than the corresponding orders in $c_{1}, \ldots, c_{p+q-m}$, so that the deformation would be the genotype of the map Expan. 
Then possibly one could show the independence of the genotype from the degrees $p, q$, at least in the case of quasi-homogeneous singularities. The details are technically very complicated even in the case $m=2$. The authors are convinced that the local degree of the map Expan for a quasihomogeneous singularity $\phi \sim t^{m}, \psi \sim t^{n}$ is equal to $(m+n-1) ! / m ! n$ ! (compare (1.8)). In general, for a curve with multiple singular points, all of them being quasi-homogeneous, a fair conjecture is that the multiplicity of Expan is the product of the local multiplicities corresponding to individual singular points.

3. Global properties of the map Expan. We begin by introducing a $\mathbb{C}^{*}$-action on the spaces Curv and Puis,

$$
a_{j} \mapsto \lambda^{j} a_{j}, \quad b_{j} \mapsto \lambda^{j} b_{j}, \quad c_{i} \mapsto \lambda^{i} c_{i} .
$$

This action is induced by the automorphism of the parameter space and target space $\phi \mapsto \lambda^{p} \phi(t / \lambda), \psi \mapsto \lambda^{q} \psi(t / \lambda)$.

Lemma 3.1. The map Expan is equivariant with respect to these actions.

The next lemma describes curves with a very special Puiseux expansion at infinity.

Lemma 3.2. A curve $\xi_{0} \in \mathrm{Curv}$ has the Puiseux expansion

$$
y=x^{q / p}+\alpha x^{(1-p) / p}+O\left(x^{-1}\right),
$$

i.e. $c_{1}=\cdots=c_{p+q-2}=0, c_{p+q-1}=\alpha$, if and only if

$$
R(t):=p \phi \dot{\psi}-q \psi \dot{\phi} \equiv p(1-q-p) \alpha ;
$$

in particular, if $\alpha=0$ then $\xi=\left(\omega^{p_{1}}, \omega^{q_{1}}\right)$, where $\omega$ is a polynomial of degree $p^{\prime}=\operatorname{gcd}(p, q)$, and for $\alpha \neq 0$ the curve is non-singular.

Proof. Consider the function

$$
H(t)=\psi(t) \phi^{-q / p}(t) .
$$

Its derivative has the form

$$
H^{\prime}(t)=\frac{1}{p} R(t) \phi^{-q / p-1}(t) .
$$

The property (3.2) means that $H(t)=1+\alpha t^{1-p-q}+\cdots$ as $t \rightarrow \infty$. Hence $H^{\prime}=(1-p-q) \alpha t^{-p-q}+\cdots \sim(1-p-q) \alpha \phi^{-q / p-1}$. From this the first statement of the lemma follows immediately.

If $\alpha=0$, then $R(t)=0$, so $H(t) \equiv 1$, i.e. $\psi^{q}=\phi^{p}$. If $\alpha \neq 0$, then $R(t)$ has no zeros, so $\dot{\phi}$ and $\dot{\psi}$ cannot have common zeros.

Recall that the non-properness set $S(G)$ of a map $G: X \rightarrow Y$ consists of the points $y \in Y$ where the number of preimages $G^{-1}(y)$ jumps down (see 
Definition 1.6). If $X=Y=\mathbb{C}^{n}$ and $G$ is a polynomial mapping, then $S(G)$ is either empty or a union of so-called ruled hypersurfaces (see [Jel]).

Proof of Theorem 1.7. In case $p^{\prime}:=\operatorname{gcd}(p, q)=1$ we need to prove two things: that the map $\operatorname{Expan}_{p, q}$ is proper, and next that its degree is equal to $(p+q-1) ! / p ! q !$. The properness follows from the $\mathbb{C}^{*}$-equivariance and the fact that $\operatorname{Expan}^{-1}(0)=0$ (by Lemmas 3.1 and 3.2). Indeed, if $|\xi| \rightarrow \infty$, then $|\operatorname{Expan}(\xi)| \rightarrow \infty$, where $|\cdot|$ are some quasi-norms compatible with the $\mathbb{C}^{*}$-action.

To calculate the degree of Expan in case $p^{\prime}=1$ we compute the number of preimages of the point $c^{*}=(0, \ldots, 0,1)$. By Lemma 3.2, $c^{*}$ is a regular value of Expan. Write $\phi=\prod\left(t-t_{i}\right), \psi=\prod\left(t-s_{j}\right), \sum t_{i}=0$ (by (1.3)). As in the proof of Lemma 3.2 the condition $\operatorname{Expan}(\xi)=c^{*}$ means that $H=1+t^{1-p-q}+\cdots$, or

$$
\log H=\sum \log \left(1-\frac{s_{j}}{t}\right)-\frac{q}{p} \sum \log \left(1-\frac{t_{i}}{t}\right)=t^{1-p-q}+\cdots .
$$

From (3.4) we get the algebraic system

$$
\begin{aligned}
\sum s_{j}-\frac{q}{p} \sum t_{i} & =0, \\
& \vdots \\
\sum s_{j}^{p+q-2}-\frac{q}{p} \sum t_{i}^{p+q-2} & =0, \\
\sum s_{j}^{p+q-1}-\frac{q}{p} \sum t_{i}^{p+q-1} & =1 .
\end{aligned}
$$

Together with $\sum t_{i}=0$ we get $p+q$ equations with $p+q$ unknowns. The product of degrees is $(p+q-1)$ !. Since there are no solutions at infinity by Lemma 3.2 and all solutions are of multiplicity one, we get $(p+q-1)$ ! finite solutions. Moreover, for any solution, $t_{i_{1}} \neq t_{i_{2}}$ and $s_{j_{1}} \neq s_{j_{2}}$ by (3.3). Dividing by the symmetry group $\Sigma(p) \times \Sigma(q)$, we find the degree of Expan.

The second statement of Theorem 1.7 is that for $p^{\prime}>1$ the non-properness set $S$ (Expan) equals $\left\{c_{1}=0\right\}$.

Consider a family $\{\widetilde{\xi} \circ \omega\}$ where $\widetilde{\xi} \in \operatorname{Curv}_{p_{1}, q_{1}}\left(p_{1}=p / p^{\prime}, q_{1}=q / p^{\prime}\right)$ is fixed and $\omega(t)=t^{p^{\prime}}+\gamma_{2} t^{p-2}+\cdots$ has varying coefficients $\gamma_{2}, \ldots, \gamma_{p^{\prime}}$. This family is non-compact, but it is sent to one point in Puis. This shows that the map Expan for $p^{\prime}>1$ is not proper.

We know that $S$ (Expan) $\neq \emptyset$ and that this is a hypersurface in Curv. On the other hand, the hypersurface $\left\{c_{1}=0\right\}$ is the bifurcation hypersurface for the number of double points of the curve $C=\xi(\mathbb{C})$. Namely, if $c_{1} \neq 0$, then a generic curve has $\frac{1}{2}\left[(p-1)(q-1)-\left(p^{\prime}-1\right)\right]$ finite double points. A generic curve with $c_{1}=0$ has at most $\frac{1}{2}\left[(p-1)(q-1)-2\left(p^{\prime}-1\right)\right]$ such points, so some double points tend to infinity as $c_{1} \rightarrow 0$ (see [BZ1]). 
Lemma 3.3. If an algebraic family $\left\{\xi_{\mu}\right\}, \mu \in \mathbb{C} \backslash\{0\}$, has $\left|\xi_{\mu}\right| \rightarrow \infty$, but $\operatorname{Expan}\left(\xi_{\mu}\right) \rightarrow c_{0} \in$ Puis $($ as $\mu \rightarrow 0)$, then there are some double points of the curve $C_{\mu}=\xi_{\mu}(\mathbb{C})$ tending to infinity.

Proof. Let $\xi_{\mu}=\left(\phi_{\mu}, \psi_{\mu}\right), \phi_{\mu}=\prod\left(t-t_{i}(\mu)\right), \psi_{\mu}=\prod\left(t-s_{j}(\mu)\right)$, where some roots of $\phi_{\mu}, \psi_{\mu}$ tend to infinity. We distinguish the collection of roots with fastest escape to infinity (by algebraicity of $\xi$ this is possible). We assume that

$$
t_{1}(\mu) \sim T_{1} \mu^{-A}, \ldots, t_{r} \sim T_{r} \mu^{-A}, s_{1} \sim S_{1} \mu^{-A}, \ldots, s_{w} \sim S_{w} \mu^{-A},
$$

$A>0$ and $t_{j}(\mu) \mu^{A}, s_{i}(\mu) \mu^{A} \rightarrow 0$ for other roots. After renormalisation of time by $t=T \mu^{-A}$, a division of $x$ and $y$ by a suitable power of $\mu$, and letting $\mu \rightarrow 0$, we obtain a curve of the form

$$
T \rightarrow\left(T^{p-r} \prod_{i=1}^{r}\left(T-T_{i}\right), T^{q-w} \prod_{j=1}^{w}\left(T-S_{j}\right)\right) .
$$

Moreover, we have $(p-r)+(q-w)>0$.

Lemma 3.4. The limit curve (3.5) has at least one double point corresponding to $T=T_{0}$ and $T=S_{0}, T_{0} S_{0} \neq 0$.

Proof. We use the Zaidenberg-Lin theorem [ZaLi]. If the curve (3.5) did not have any double points, it could be reduced to the form $x=T^{k}, y=T^{l}$ with $\operatorname{gcd}(k, l)=1$. Since the ratio $q / p=q_{1} / p_{1}$ is not an integer, the curve (3.5) cannot be simplified further. Since, additionally, $\operatorname{gcd}(p, q) \neq 1$, the lemma follows.

From Lemma 3.4 it follows that as $\mu \rightarrow 0$ some pair, say $t_{0}(\mu)=T_{0} \mu^{-A}$, $s_{0}(\mu)=S_{0} \mu^{-A}$, representing a double point of $C_{\mu}, \xi\left(t_{0}(\mu)\right)=\xi\left(s_{0}(\mu)\right)$ tends to infinity. This implies a bifurcation of double point at $\mu=0$. By Lemma 3.3 we get $c_{1}(\mu) \rightarrow 0$ as $\mu \rightarrow 0$. Therefore $S($ Expan $) \subset\left\{c_{1}=0\right\}$.

There remains the last statement of Theorem 1.7, namely the calculation of $\operatorname{deg}_{\text {top }}$ Expan in the case $p^{\prime}=2$. We shall prove the following general formula:

Theorem 3.5. If $p^{\prime}=2$ and $c_{1}=\cdots=c_{2 k-1}=0 \neq c_{2 k+1}$, then $\operatorname{Expan}^{-1}(c)$, where $c=\left(c_{1}, \ldots, c_{p+q-1}\right)$, consists of

$$
\frac{(p+q-1) !}{p ! q !}-\frac{2 k+1}{2}\left(\operatorname{deg}_{\text {top }} \operatorname{Expan}_{p_{1}, q_{1}}\right)^{2}
$$

points counted with multiplicities.

Proof. Assume that $q_{1}=q / p^{\prime}$ is odd (the case of $p_{1}$ odd is analogous). Consider the power series

$$
\phi(t)^{k / p}=t^{k}+\alpha_{k, k-1} t^{k-1}+\alpha_{k, k-2} t^{k-2}+\cdots,
$$


where $\alpha_{k, j}=\alpha_{k, j}(a)$ are polynomials in the coefficients $a_{i}$ of $\phi(t)$, or equivalently, symmetric homogeneous polynomials of degree $k-j$ of the roots $t_{1}, \ldots, t_{p}$. For given Puiseux coefficients $c \in$ Puis the preimages $\operatorname{Expan}^{-1}(c)$ correspond to solution to the system of algebraic equations

$$
\begin{aligned}
\alpha_{q,-1}+c_{1} \alpha_{q-1,-1}+\cdots+c_{p+q-1} \alpha_{1-p,-1} & =0, \\
\alpha_{q,-2}+c_{1} \alpha_{q-1,-2}+\cdots+c_{p+q-1} \alpha_{1-p,-2} & =0 \\
& \vdots \\
\alpha_{q,-2}+c_{1} \alpha_{q-1,-1}+\cdots+c_{p+q-1} \alpha_{1-p,-2} & =0 .
\end{aligned}
$$

Indeed, if $a_{i}$ satisfy this equation, then after putting $b_{k}=\alpha_{q, k}+c_{1} \alpha_{q-1, k}+$ $\cdots+c_{p+q-1} \alpha_{1-p, k}$ we get a curve $\xi=(\phi, \psi)$ such that $\operatorname{Expan}(\xi)=c$.

We will treat (3.7) as a system of equations in the roots $t_{1}, \ldots, t_{p}$ of $\phi(t)$. The product of degrees is $(q+1) \ldots(q+p-1)$. The symmetry group $\Sigma(p)$ acts on the solutions, so the number of solutions modulo the symmetry equals $(p+q-1) ! / p ! q$ !. But some solutions are at infinity, therefore we have to compute the number (with multiplicities) of solutions to (3.7) at infinity.

Let us introduce homogeneous coordinates $\left[t_{0}: t_{1}: \cdots: t_{p}\right]$, with the condition $t_{1}+\cdots+t_{p}=0$. The homogenisation of (3.7) relies on substituting $c_{i}$ with $c_{i} t_{0}^{i}$.

Then solutions at infinity correspond to $t_{0}=0$, or equivalently, to $c_{1}=$ $\cdots=c_{p+q-1}=0$. Lemma 3.2 implies $\phi=\chi^{p_{1}}, \psi=\chi^{q_{1}}$ for a quadratic polynomial $\chi\left(\right.$ since $\left.p^{\prime}=2\right)$, which can be rescaled to be

$$
\chi=(t-1)(t+1) .
$$

This corresponds to $\frac{1}{2}\left(\begin{array}{c}2 p_{1} \\ p_{1}\end{array}\right)$ points $\left[0: \nu_{1}: \cdots: \nu_{p}\right]$ with $\nu_{i}= \pm 1$ and $\sum \nu_{i}=0$. Theorem will be proved once we show that the multiplicity of (3.7) at each such point is equal to $(2 k+1)\left[\left(q_{1}+1\right) \ldots\left(q_{1}+p_{1}-1\right)\right]^{2}$.

Pick $P_{0}=[0: 1: \cdots: 1:-1: \cdots:-1]$ as one such point. Introduce local coordinates $x_{i}=t_{i} / t_{2 p_{1}}, i=0, \ldots, 2 p-1, t_{2 p_{1}}=0$ around $P_{0}$ as follows:

$$
\begin{cases}x_{0}=\gamma, & \text { for } i=1, \ldots, p_{1}, \\ x_{i}=-1+\delta_{i} & \text { for } i=p_{1}+1, \ldots, 2 p_{1}-1, \\ x_{i}=1+\varepsilon_{i} & \\ \sum \delta_{i}+\sum \varepsilon_{i}=0 . & \end{cases}
$$

Then we have

$$
\phi=\prod_{i=1}^{2 p_{1}}\left(t-t_{i}\right)=\prod_{i=1}^{2 p_{1}}\left(t+x_{i}\right)=\left(t^{2}-1\right)^{2 p_{1}} \prod\left(1+\frac{\delta_{i}}{t-1}\right) \prod\left(1+\frac{\varepsilon_{i}}{t+1}\right) .
$$

We compute the leading terms in $\delta, \varepsilon$ in the expansion of the polynomials 
$\alpha_{k,-j}$ from (3.6). If $k=2 k_{1}$, then

$$
\phi^{k / p}=\left(t^{2}-1\right)^{k_{1}} \prod\left(1+\frac{\delta_{i}}{t-1}\right)^{k_{1} / p_{1}} \prod\left(1+\frac{\varepsilon_{i}}{t+1}\right)^{k_{1} / p_{1}} .
$$

We can easily see that the degree of the leading term is equal to $k_{1}+1$. In fact, the poles at $t=1$ of the expansion

$$
\left(1+\frac{\delta_{i}}{t-1}\right)^{k_{1} / p_{1}}=1+\frac{k_{1}}{p_{1}} \frac{\delta_{i}}{t-1}+\cdots
$$

cancel with $\left(t^{2}-1\right)^{k_{1}}$. The same holds for $\varepsilon$. In particular

$$
\begin{aligned}
& \alpha_{2 k_{1},-1}=\left(\begin{array}{c}
k_{1} / p_{1} \\
k_{1}+1
\end{array}\right)\left[\sum \delta_{i}^{k_{1}+1}+\sum \varepsilon_{i}^{k_{1}+1}\right]+\cdots, \\
& \alpha_{2 k_{1},-2}=\left(\begin{array}{c}
k_{1} / p_{1} \\
k_{1}+1
\end{array}\right)\left[\sum \delta_{i}^{k_{1}+1}-\sum \varepsilon_{i}^{k_{1}+1}\right]+\cdots .
\end{aligned}
$$

The dots denote terms of higher order in $\varepsilon, \delta$. The symbol $\left(\begin{array}{l}a \\ n\end{array}\right)$ with $n \in \mathbb{N}$, $a \in \mathbb{R}$ should be read as $a(a-1)(a-2) \ldots(a-n+1) / n$ !. We have also used $\frac{1}{t \pm 1}=\frac{1}{t} \mp \frac{1}{t^{2}}+\cdots$.

Next $\Delta^{(1)} \alpha_{2 k_{1},-1}=\alpha_{2 k_{1},-1}-\alpha_{2 k_{1},-3}$ is the coefficient of $t^{-1}$ in the expansion of $\left(t^{2}-1\right) \phi^{k / p}$. We find that

$$
\begin{aligned}
& \Delta^{(1)} \alpha_{2 k_{1},-1}=\text { const } \cdot\left[\sum \delta_{i}^{k_{1}+2}+\sum \varepsilon_{i}^{k_{1}+2}\right]+\cdots, \\
& \Delta^{(1)} \alpha_{2 k_{1},-2}=\text { const } \cdot\left[\sum \delta_{i}^{k_{1}+2}-\sum \varepsilon_{i}^{k_{1}+2}\right]+\cdots .
\end{aligned}
$$

Generally,

$$
\begin{aligned}
& \Delta^{(j)} \alpha_{2 k_{1},-1}=\text { const } \cdot\left[\sum \delta_{i}^{k_{1}+j+1}+\sum \varepsilon_{i}^{k_{1}+j+1}\right]+\cdots \\
& \Delta^{(j)} \alpha_{2 k_{1},-2}=\text { const } \cdot\left[\sum \delta_{i}^{k_{1}+j+1}-\sum \varepsilon_{i}^{k_{1}+j+1}\right]+\cdots \\
& \Delta^{(j)} \alpha_{2 k_{1},-3}=\text { const } \cdot\left[\sum \delta_{i}^{k_{1}+j+1}+\sum \varepsilon_{i}^{k_{1}+j+1}\right]+\cdots,
\end{aligned}
$$

where $\Delta^{(j)} \alpha_{2 k_{1},-i}=\alpha_{2 k_{1},-i}-\left(\begin{array}{l}j \\ 1\end{array}\right) \alpha_{2 k_{1},-i-2}+\left(\begin{array}{l}j \\ 2\end{array}\right) \alpha_{2 k_{1},-i-4}+\cdots \pm \alpha_{2 k_{1},-i-2 j}$ corresponds to the expansion of $\left(t^{2}-1\right)^{j} \phi^{k / p}$. The leading terms in (3.7) are linearly dependent, but the leading terms of $\Delta^{(j)}$ are not.

If $k=2 k_{1}+1$ is odd then

$$
\phi^{k / 2 p_{1}}=t\left(1-\frac{1}{t^{2}}\right)^{1 / 2}\left[\left(t^{2}-1\right)^{2 k_{1}} \prod\left(1+\frac{\delta_{i}}{t-1}\right)^{k / p} \prod\left(1+\frac{\varepsilon_{i}}{t+1}\right)^{k / p}\right] .
$$

We see that

$$
\begin{aligned}
\alpha_{k,-(2 i+1)} & =(-1)^{i}\left(\begin{array}{c}
1 / 2 \\
i-1
\end{array}\right), \\
\alpha_{k,-2 i} & =O(\delta, \varepsilon) .
\end{aligned}
$$


The system (3.7) takes now the following algebraic form:

$$
\begin{aligned}
& \alpha_{q,-1}+c_{1} \gamma \alpha_{q-1,-1}+\cdots=0, \\
& \alpha_{q,-2}+c_{1} \gamma \alpha_{q-1,-2}+\cdots=0,
\end{aligned}
$$

$$
\alpha_{q,-2}+c_{1} \gamma \alpha_{q-1,-1}+\cdots=0 .
$$

If $c_{1} \neq 0$ then subtracting $(3.14)_{1}$ from $(3.14)_{3}$ we get $\gamma=$ const. $\Delta^{(1)} \alpha_{2 q_{1},-1}+\cdots$, so $\gamma \sim O\left((\delta, \varepsilon)^{2 q_{1}+2}\right)$. (If $c_{1}=\cdots=c_{2 k-1}=0 \neq c_{2 k+1}$ then we obtain $\gamma^{2 k+1}=$ const. $\Delta^{(1)} \alpha_{2 q_{1},-1}$ in the obvious way.) We substitute this $\gamma\left(\right.$ or $\left.\gamma^{2 k+1}\right)$ to the equation $(3.14)_{2},(3.14)_{3}, \ldots$ The leading terms in all these equations are $\alpha_{2 q_{1},-i}(\delta, \varepsilon)$ and are all of order $q_{1}+1$. But then we make linear combinations of odd and even equations, i.e. $(3.14)_{3}-(3.14)_{5}$, $(3.14)_{3}-2 \cdot(3.14)_{5}+(3.14)_{7}, \ldots$ and $(3.14)_{2}-(3.14)_{4},(3.14)_{2}-2 \cdot(3.14)_{4}+$ $(3.14)_{6}, \ldots$, to get a system of equations with leading terms $\Delta_{2 q_{1},-3}^{(j)}$ and $\Delta_{2 q_{1},-2}^{(j)}$. The homogeneous part of these leading terms is proportional to $\sum \delta_{i}^{q_{1}+j+1} \pm \sum \varepsilon_{i}^{q_{1}+j+1}$ (see (3.11)). It is not difficult to see that $\delta_{i}=\varepsilon_{i}=0$ is the only solution, with multiplicity being the product of the degrees

$$
\left(q_{1}+1\right) \ldots\left(q_{1}+p_{1}-1\right) \times\left(q_{1}+1\right) \ldots\left(q_{1}+p_{1}-1\right) .
$$

Together with the first equation

$$
\text { const } \cdot c_{2 k+1} \gamma^{2 k+1}+\alpha_{2 q_{1},-1}(\delta, \varepsilon)
$$

we get the required formula.

The proof of Theorem 1.7 is now complete.

By now we do not know how to calculate the degree of Expan in the general case, the major problem being that the locus at infinity, which is zero-dimensional if $p^{\prime}=2$, has positive dimension in the general case. The general intersection theory (see [Ful]) should be helpful, but again details are very complicated.

We finish this work with the following two interesting results:

Proposition 3.6. We have $c_{q+p}=c_{q+2 p}=\cdots=0$.

Proof. We have

$$
c_{p+q}=\frac{1}{2 \pi i} \int_{|\tau|=R} y(\tau) \tau^{p-1} d \tau, \quad R \rightarrow \infty,
$$

where $\tau=\phi(t)^{1 / p}=t(1+\cdots)$ and $y(\tau)=\psi(t)$. Replacing $\tau$ by $t$ with $\frac{d \tau}{d t}=\left(1+\frac{p-2}{p} a_{2} t^{-1}+\cdots+\frac{1}{p} a_{p-1} t^{1-p}\right) \cdot\left(1+a_{2} t^{-2}+\cdots+a_{p} t^{-p}\right)^{1 / p-1}$, we get 


$$
c_{p+q}=\frac{1}{2 \pi i} \int_{|t|=R} \psi(t)\left(1+\cdots+a_{p-1} p t^{1-p}\right) t^{p-1} d t=0 .
$$

Analogously we prove the vanishing of $c_{q+j p}$.

It is natural to ask about the dependence of the self-intersection points of $C=\xi(\mathbb{C})$ on $\xi$, i.e. on $(a, b) \in \mathbb{C}^{p+q-1}$. The preimages of such points are pairs $\left(t_{i}, s_{i}\right), i=1, \ldots, \delta_{\max }$, such that $\xi\left(t_{i}\right)=\xi\left(s_{i}\right)$. We have $2 \delta_{\max }=$ $(p-1)(q-1)-\left(p^{\prime}-1\right)$ algebraic functions $t_{i}(a, b), s_{i}(a, b)$. The number of these functions is larger than the number of parameters. Moreover, the functions are invariant with respect to the changes

$$
(x, y) \mapsto(x+\alpha, y+P(x)), \quad \operatorname{deg} P<q / p .
$$

So, we ask which collections of $N=p+q-3-[q / p]$ elements from the set $\left(t_{1}, s_{1}, \ldots, t_{\delta_{\max }}, s_{\delta_{\max }}\right)$ form local parameters in the space Curv/Eq, where $\mathrm{Eq}$ is the the group of changes (3.15) (we can identify the space Curv/Eq with the subset of $\operatorname{Curv}_{p, q}$ given by $\left.b_{q-k p}=0, b_{q}=0, a_{p}=0\right)$. Any such collection consists of $k$ pairs and $p+q-3-[q / p]-2 k$ singles; after some renumbering it is of the form

$$
t_{1}, s_{1}, \ldots, t_{k}, s_{k}, t_{k+1}, \ldots, t_{N-k} .
$$

We have the following result which we present without proof

Proposition 3.7. If $k \leq p-2$, then the functions (3.16) form a local coordinate system near a typical point in Curv/Eq.

EXAmple 3.8. Assume $\phi(t)=t^{2}$ and $\psi=t^{q}+b_{1} t^{q-1}+\cdots+b_{q-1} t$, $q=2 l+1$. The preimages of double points are $t_{i}, t_{i}-1$, where $t_{i}^{2}$ are roots of the polynomial $\lambda^{l}+b_{2} \lambda^{l-1}+\cdots+b_{2 l}$. It is clear that $t_{1}, \ldots, t_{l}$ are independent functions of $b_{2}, \ldots, b_{2 l}$.

\section{References}

[AVG] V. I. Arnold, A. N. Varchenko and S. M. Gusein-Zade, Singularities of Differentiable Mappings, Vol. I, Monogr. Math. 82, Birkhäuser, Boston, 1985; Russian original: Nauka, Moscow, 1982.

[BZ1] M. Borodzik and H. Żołądek, Complex algebraic plane curves via Poincaré-Hopf formula. I. Parametric lines, Pacific J. Math. 229 (2007), 307-338.

[BZ2] -, 一, Complex algebraic plane curves via Poincaré-Hopf formula. III. Codimension bounds, preprint, Warsaw Univ., 2007.

[Ful] W. Fulton, Intersection Theory, Springer, Berlin, 1998.

[Jel] Z. Jelonek, The set of points at which a polynomial map is not proper, Ann. Polon. Math. 58 (1993) 259-266.

[Lan] S. K. Lando, Ramified coverings of the two dimensional sphere and intersection theory in the space of meromorphic functions, Uspekhi Mat. Nauk 57 (2002), 29-98 (in Russian); English transl.: Russian Math. Surveys 57 (2002), 463-533. 
[Mor] B. Morin, Formes canoniques des singularités d'une application différentiable, C. R. Acad. Sci. Paris 260 (1965), 5662-5665, 6503-6506.

[War] B. L. van der Werden, Algebra I, Springer, Berlin, 1971.

[ZaLi] M. G. Zaidenberg and V. Ya. Lin, An irreducible, simply connected algebraic curve in $\mathbb{C}^{2}$ is equivalent to a quasi-homogeneous curve, Dokl. Akad. Nauk SSSR 271 (1983), 1048-1052 (in Russian).

[Zol] H. Żołądek, Application of Newton-Puiseux charts in the Jacobian problem, Topology, in press.

Institute of Mathematics

University of Warsaw

Banacha 2

02-097 Warszawa, Poland

E-mail: mcboro@mimuw.edu.pl

zoladek@mimuw.edu.pl

Received 10.12.2007

and in final form 18.2.2008 